\title{
Clifford Algebra of Points, Lines and Planes
}

\author{
J.M. Selig \\ School of Computing, Information Systems \& Mathematics \\ South Bank University \\ London SE1 0AA, U.K.
}

\begin{abstract}
The Clifford algebra for the group of rigid body motions is described. Linear elements, that is points, lines and planes are identified as homogeneous elements in the algebra. In each case the action of the group of rigid motions on the linear elements is found. The relationships between these linear elements are found in terms of operations in the algebra. That is, incidence relations, the conditions for a point to lie on a line for example are found. Distance relations, like the distance between a point and a plane are found. Also the meet and join of linear elements, for example, the line determined by two planes and the plane defined by a line and a point, are found. Finally three examples of the use of the algebra are given: a computer graphics problem on the visibility of the apparent crossing of a pair of lines, an assembly problem concerning a double peg-in-hole assembly, and a problem from computer vision on finding epipolar lines in a stereo vision system.
\end{abstract}

\section{Introduction}

Many problems in robotics are essentially problems in Euclidean geometry. This is true for both robot vision and kinematics. We often need to find the intersection of a plane and a line or the line determined by a pair of points. In this work a Clifford algebra is described in which most of these problems can be handled very simply. Points, lines and planes are described by homogeneous elements of the algebra and geometric relations between these elements are represented by simple formulas in the algebra.

Although all of the Euclidean geometry that we describe can be done by other means the aim of this paper is to describe a unifying algebraic framework for the geometry. If the set-up is simple enough then many results can be done symbolically and often by hand. The symbolic point of view has many uses.

For example, many workers have suggested that computer drafting systems should have some symbolic capability. This would enable them to store symbolic relationships between parts, and hence be able to exclude infeasible configurations, see [1].

Looking ahead to the example in section 5.3, suppose a stereo vision system had movable cameras as in many tele-presence devices currently under development. It would be sensible to store the relations for the epipolar line in a symbolic form, so that in a particular position the relevant line could be computed by simple substitution. 
In robot kinematics, we often require symbolic solutions to the inverse kinematics problem. The Clifford algebra presented here seems to give an efficient way to derive such formulas when they exist.

In a paper by Ruiz and Ferreira [2], formulas were derived for the distances between points and planes, points and lines and so forth. The main aim of that work seems to have been the solution of problems involving several of these geometrical constraints using the technique of Gröbner bases. However, the derivation of the geometrical formulas was rather cumbersome and the representation of the linear elements, the points lines and planes was somewhat arbitrary.

In this work the relations between linear elements in space is studied in some detail, that is the distances between points, lines and planes, conditions for incidence and so on. By introducing the Clifford algebra we are able to describe the possible relations in a systematic way, so that, for example the condition for incidence between a pair of linear elements is given by a single equation no matter whether the elements are points, lines or planes. Moreover, these relations are rather simple in form and hence the system of equations that would be generated by sets of geometric constraints are in general, simpler than those found in [2].

Several other attempts have been made to introduce Clifford algebras into engineering, notably Lasenby et al [3]. This work concentrates on the Clifford algebra associated with the group of rotations. This means that lines and planes which do not pass through the origin cannot be incorporated in a natural way. By contrast, this work follows Porteous [4] in looking at a Clifford algebra naturally associated with the group of rigid body motions. These ideas in fact can be traced back to Clifford himself who introduce what he called "biquaternions" to model the group of rigid body motions, [5].

The rest of this paper is organised as follows. The next section introduces the Clifford algebra, showing how the group of rigid body motions can be identified with a subset of the algebra. The following section introduces the linear elements, the points, lines and planes, showing which algebra elements correspond to these geometric objects. Then follows a section which deals with the relationships between the linear elements. This is done on a case by case basis, but a final subsection summarises the result derived. Finally, three examples are given, a computer graphics problem on the visibility of the apparent crossing of a pair of lines, an assembly problem concerning a double peg-in-hole assembly, and a problem from computer vision on finding epipolar lines in a stereo vision system.

\section{The Clifford Algebra}

As mentioned above the treatment here closely follows Porteous [4, p.276], and used in [6, Chap. 9]. A Clifford algebra is an associative algebra generated by a number of basis elements $e_{1}, e_{2}, \ldots, e_{n}$. The product of algebra elements is simply denoted by juxtaposition, for example the product of a pair of generators is written $e_{1} e_{2}$. This is a new element of the algebra and we can produce more elements with more products. However, the products of generators are subject to a few relations. First of all the 
product of generator elements is anti-commutative,

$$
e_{i} e_{j}=-e_{j} e_{i}, \quad \text { for all } i \neq j
$$

The second set of relations concerns the squares of the generators. In standard approaches to Clifford algebra the square of a generator elements is always -1 , see for example [3], however, it is also possible to require that generators square to 0 or +1 . Here we will consider an algebra which has 3 generators $e_{1}, e_{2}, e_{3}$ which square to -1 and a single generator $e$ which squares to 0 ,

$$
e_{1}^{2}=e_{2}^{2}=e_{3}^{2}=-1, \quad e^{2}=0
$$

Thus the algebra has $2^{4}=16$ basis elements,

$$
\begin{gathered}
1 \\
e_{1}, e_{2}, e_{3}, e \\
e_{1} e_{2}, e_{1} e_{3}, e_{1} e, e_{2} e_{3}, e_{2} e, e_{3} e \\
e_{1} e_{2} e_{3}, e_{1} e_{2} e, e_{1} e_{3} e, e_{2} e_{3} e \\
e_{1} e_{2} e_{3} e
\end{gathered}
$$

A general element of the algebra is a linear combination of these basis elements. For example, $1+e_{3}$ or $e_{2}+2 e_{1} e_{3}$. The product of two such elements can be simplified by applying the distributive rule, the anti-commutation properties and relations for the squares of generators,

$$
\begin{aligned}
\left(1+e_{3}\right)\left(e_{2}+2 e_{1} e_{3}\right) & =e_{2}+2 e_{1} e_{3}+e_{3} e_{2}+2 e_{3} e_{1} e_{3} \\
& =e_{2}+2 e_{1} e_{3}-e_{2} e_{3}-2 e_{1} e_{3} e_{3} \\
& =e_{2}+2 e_{1} e_{3}-e_{2} e_{3}+2 e_{1}
\end{aligned}
$$

Notice that each basis element has a grade corresponding to the number of generators it contains. So this algebra contains one basis element of grade 0 , four of grade 1 (the generators), six of grade 2, four of grade 3 and one of the highest grade 4. Algebra elements which consist of linear combinations of basis elements all of the same grade are called homogeneous elements. In the following, subspaces of homogeneous elements play a major role.

Finally here, we define an operation called conjugation on the algebra. Conjugation is the Clifford algebra generalisation of complex conjugation and like complex conjugation it plays a central role in the algebra. It usually turns up when we are trying to produce a scalar quantity, just like the modulus of a complex number. However, in the Clifford algebra here, its use is more extensive because it can be used to produce expressions which behave covariantly with respect to the action of the group of rigid body motions.

The conjugation operation is a linear mapping of the algebra to itself. The conjugation of an element will be denoted by an asterisk, so for example $e_{1}^{*}=-e_{1}$. In fact the conjugate of any generator is simply the negative of the generator. The conjugate of other basis elements can be found by conjugating the individual generators 
and reversing their order. For example, $\left(e_{1} e_{2}\right)^{*}=\left(-e_{2}\right)\left(-e_{1}\right)=-e_{1} e_{2}$. A three generator example would be, $\left(e_{1} e_{2} e_{3}\right)^{*}=\left(-e_{3}\right)\left(-e_{2}\right)\left(-e_{1}\right)=e_{1} e_{2} e_{3}$. The conjugate of any element of the algebra can now be found by using the linearity property of the operation. That is, the conjugate of a general element of the algebra is found by conjugating the generators. For example, if $a=e_{1}+3 e_{1} e_{2}$ then the conjugate of $a$ will be, $a^{*}=-e_{1}-3 e_{1} e_{2}$.

Grade 0 elements, (scalars) are required to be unchanged by conjugation. Notice that this is consistent with our previous definitions,

$$
1^{*}=\left(-e_{1} e_{1}\right)^{*}=-e_{1}^{*} e_{1}^{*}=-e_{1} e_{1}=1
$$

It is not difficult to see that for any pair of elements $a$ and $b$ in the algebra, the conjugate of the product is given by, $(a b)^{*}=b^{*} a^{*}$.

\subsection{The Group of Rigid Body motions}

The reason why this Clifford algebra is so important is that it contains the the group of rigid body motions in 3-D, actually its double cover. It also contains the Lie algebra of the group and several geometrical representations. That is, the spaces of points lines and planes in 3-D which are transformed by the group of rigid body motions.

Here we look at the group of rigid body motions. The group elements are elements of the algebra which have the shape,

$$
g=h+\frac{1}{2} \text { the }
$$

The translation part of the rigid body motion corresponds to,

$$
t=t_{x} e_{1}+t_{y} e_{2}+t_{z} e_{3}
$$

where $t_{x}, t_{y}$ and $t_{z}$ are the $x, y$ and $z$-components of the translation vector.

The rotational part of the motion is given by,

$$
h=\cos (\theta / 2)+\sin (\theta / 2)\left(v_{x} e_{2} e_{3}+v_{y} e_{3} e_{1}+v_{z} e_{1} e_{2}\right)
$$

where $\theta$ is the angle of rotation and $v_{x}, v_{y}$ and $v_{z}$ are the components of the unit vector along the axis of the rotation. Notice that these elements satisfy the condition, $h h^{*}=$ $h^{*} h=1$ since $v_{x}^{2}+v_{y}^{2}+v_{z}^{2}=1$. This representation of the rotation group is equivalent to the usual quaternion representation, to see this simply replace the basis elements $e_{2} e_{3}, e_{3} e_{1}$ and $e_{1} e_{2}$ by the unit quaternions $i, j$ and $k$ respectively.

In fact the group of element defined above double cover the rotations since both $h$ and $-h$ correspond to the same rotation. This is probably best understood by considering the action of these group elements on points. Let a point in $\mathbb{R}^{3}$ be represented by Clifford algebra element of the form, $r=x e_{1}+y e_{2}+z e_{3}$. The action of the rotation group on these points can now be written as,

$$
r^{\prime}=h r h^{*}
$$


This is straightforward but tedious to check. Notice that the product of two rotations is represented by the Clifford product of the corresponding algebra elements.

We will see in later sections that this is part of a general pattern, the action of the group on any linear element is given by,

$$
\xi^{\prime}=g \xi g^{*}
$$

where $\xi$ is a point, line or plane and $g$ is an element of the group of rigid body motions.

Returning to the group of rigid body motions, we see that a pure translation is represented by an element of the form $\left(1+\frac{1}{2} t e\right)$, that is an element with rotational part $h=1$. So the product of a pure rotation $h$, with a translation is given by,

$$
h\left(1+\frac{1}{2} t e\right)=\left(h+\frac{1}{2} h t e\right)=\left(h+\frac{1}{2}\left(h t h^{*}\right) h e\right)
$$

which gives the action we would expect of a rotation on a translation.

Finally here notice that the elements of the group will satisfy the equation,

$$
g g^{*}=1
$$

This means that the inverse of a group element $g$ is given by its conjugate $g^{*}$.

A more detailed account of the above can be found in [6, Chap. 9]

\subsection{Hodge Star}

In this section a new operation on the algebra is introduced, the Hodge star. This is often used in exterior algebras as a way of introducing a metric. The Clifford algebras are very closely related to the exterior algebras. In Clifford algebras where all the generators square to -1 this operation is simply (left) multiplication by the basis element of highest grade. Hence a separate operation is unnecessary. Here though, the basis element of highest grade, $e_{1} e_{2} e_{3} e$ will not give a 1-to-1 mapping of the algebra to itself. The product with all other basis element containing the generator $e$ will give 0 . This is important because the operator we want to define is a sort of dualising operator, mapping each element to its 'dual'.

As usual, we will define this linear map by specifying its action of the basis elements of the algebra and then extend the definition to all other elements by demanding linearity. We write the Hodge star of an algebra element $a$ as $\star a$. For basis elements we require that $a(\star a)=e_{1} e_{2} e_{3} e$, thus for the basis elements we have,

$$
\begin{aligned}
\star 1 & =e_{1} e_{2} e_{3} e & \star e_{1} e_{2} e_{3} e & =1 \\
\star e_{1} & =e_{2} e_{3} e & \star e_{2} e_{3} e & =-e_{1} \\
\star e_{2} & =e_{3} e_{1} e & \star e_{3} e_{1} e & =-e_{2} \\
\star e_{3} & =e_{1} e_{2} e & \star e_{1} e_{2} e & =-e_{3} \\
\star e & =-e_{1} e_{2} e_{3} & \star e_{1} e_{2} e_{3} & =e \\
\star e_{1} e_{2} & =e_{3} e & \star e_{3} e & =e_{1} e_{2} \\
\star e_{2} e_{3} & =e_{1} e & \star e_{1} e & =e_{2} e_{3} \\
\star e_{3} e_{1} & =e_{2} e & \star e_{2} e & =e_{3} e_{1}
\end{aligned}
$$




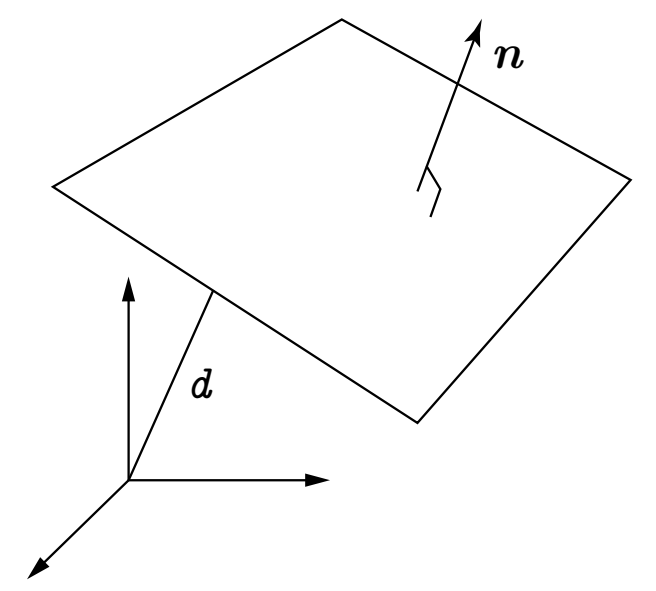

Figure 1: A Plane

So, for example, if $p=e_{1} e_{2} e_{3}+x e_{2} e_{3} e+y e_{3} e_{1} e+z e_{1} e_{2} e$ then $\star p=e-x e_{1}-y e_{2}-$ $z e_{3}$. Notice that this operation is not coordinate invariant, a change of basis will change the operation.

\section{Points, Lines and Planes}

In this section we detail the representation of points, lines and planes as elements in the Clifford algebra. Also we show how the group of rigid body motions acts on these elements.

\subsection{Planes}

We can specify a plane by giving its unit normal vector $\mathbf{n}$ and the perpendicular distance from the origin, see figure 1. As usual, the vector equation of the plane is given by,

$$
\mathbf{n} \cdot \mathbf{r}=d
$$

where $\mathbf{r}$ is any point on the plane. Notice that these are oriented planes since reversing the sign of $\mathbf{n}$ and $d$ will invert the orientation of the plane.

In the Clifford algebra we can represent planes as elements of the form,

$$
\pi=n_{x} e_{1}+n_{y} e_{2}+n_{z} e_{3}+d e
$$

These elements must satisfy the quadratic condition,

$$
\pi \pi^{*}=1
$$

this ensures that the vector $\mathbf{n}$ has unit length. Note that, $\pi^{*}=-\pi$, hence we could also write the condition as $\pi^{2}=-1$. Now if we subject the plane to a rigid body motion the 
normal vector and distance to the origin will change as follows,

$$
\mathbf{n}^{\prime}=R \mathbf{n}, \quad d^{\prime}=d+(R \mathbf{n}) \cdot \mathbf{t}
$$

This is most easily seen by considering the effect on the vector equation for the plane above. In the Clifford algebra this can be represented by,

$$
\pi^{\prime}=g \pi g^{*}
$$

as was forecast in section 2.1 Explicitly this will be,

$$
\pi^{\prime}=\left(h+\frac{1}{2} t h e\right)(n+d e)\left(h^{*}+\frac{1}{2} e h^{*} t\right)=h n h^{*}+\left(d-\frac{1}{2}\left(h n h^{*} t+t h n h^{*}\right)\right) e
$$

where we have made extensive use of the relations of the Clifford algebra.

\subsection{Points}

We will represent points in $\mathbb{R}^{3}$ by elements of the Clifford algebra of the form,

$$
p=e_{1} e_{2} e_{3}+x e_{2} e_{3} e+y e_{3} e_{1} e+z e_{1} e_{2} e
$$

The effect of a rigid body motion is given by,

$$
p^{\prime}=g p g^{*}
$$

Notice that these points satisfy the equation $p p^{*}=1$, (or $p^{2}=1$, since $p^{*}=p$ ) however, they are not the only solutions. There is another $\mathbb{R}^{3}$ of solutions where the coefficient of $e_{1} e_{2} e_{3}$ is -1 instead of +1 . We could think of these as 'negative' points, or points with the opposite orientation (whatever that means).

This representation is different from the one given in [4] and used in [6]. That representation has a slightly different group action, which would make the relations given below more complicated.

\subsection{Lines}

Lines in $\mathbb{R}^{3}$ can be specified by a pair of vectors, a unit vector $\mathbf{v}$, in the direction of the line and a moment vector $\mathbf{u}=\mathbf{r} \times \mathbf{v}$, where $\mathbf{r}$ is the position vector of any point on the line, see figure 2 . These vectors will thus be orthogonal $\mathbf{v} \cdot \mathbf{u}=0$. In the Clifford algebra we will represent a line by elements of the form,

$$
\ell=\left(v_{x} e_{2} e_{3}+v_{y} e_{3} e_{1}+v_{z} e_{1} e_{2}\right)+\left(u_{x} e_{1} e+u_{y} e_{2} e+u_{z} e_{3} e\right)
$$

but satisfying the relation,

$$
\ell \ell^{*}=1
$$

This relation combines the requirements that $\mathbf{v}$ is a unit vector and that $\mathbf{v}$ and $\mathbf{u}$ are orthogonal. These lines are in fact directed lines since $-\ell$ is the same line as $\ell$ but with the opposite direction. 


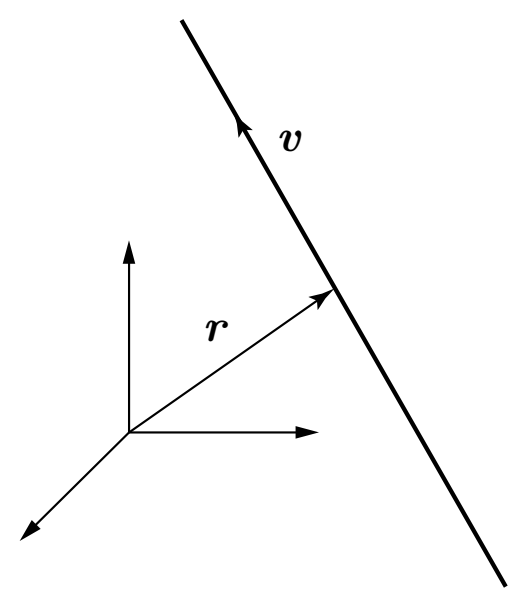

Figure 2: A Line in Space

The effect of a rigid body motion on these vectors is given by,

$$
\mathbf{v}^{\prime}=R \mathbf{v}, \quad \mathbf{u}^{\prime}=R \mathbf{u}+\mathbf{t} \times R \mathbf{v}
$$

and hence the effect of a rigid body motion on a line can be represented as,

$$
\ell^{\prime}=g \ell g^{*}
$$

\section{Relations between Points, Lines and Planes}

In this section we investigate the relations between the linear elements. There are several forms of relation to consider, conditions for incidence, distances between elements and operations which form a new element. In different cases the the relations which are appropriate will be different. There is no distance to consider if the elements intersect in the general case, like a line and a plane. Often the condition for incidence is that the distance between the elements vanishes. Moreover, the operation to construct a new element from others will usually fail if an incidence condition is satisfied, for example consider the plane formed by a point and a line, if the point lies on the line then no unique plane is defined.

\subsection{A Pair of Planes}

The simplest case to consider turns out to be a pair of planes, this is because we are representing planes by grade one elements in the Clifford algebra. So consider a pair of planes,

$$
\begin{aligned}
& \pi_{1}=n_{1 x} e_{1}+n_{1 y} e_{2}+n_{1 z} e_{3}+d_{1} e \\
& \pi_{2}=n_{2 x} e_{1}+n_{2 y} e_{2}+n_{2 z} e_{3}+d_{2} e
\end{aligned}
$$


In general, a pair of planes will determine a line. Since the line lies in both planes, it will be perpendicular to both normal vectors. Hence the direction of the line will be proportional to the vector product of the normals to the planes,

$$
\mathbf{v} \propto \mathbf{n}_{1} \times \mathbf{n}_{2}
$$

To find the moment of the line, consider a point $\mathbf{r}$ on the line. Since this point lies in both planes we have $\mathbf{n}_{1} \cdot \mathbf{r}=d_{1}$ and $\mathbf{n}_{2} \cdot \mathbf{r}=d_{2}$. Thus the moment is given by,

$$
\mathbf{r} \times \mathbf{v} \propto \mathbf{r} \times\left(\mathbf{n}_{1} \times \mathbf{n}_{2}\right)=d_{2} \mathbf{n}_{1}-d_{1} \mathbf{n}_{2}
$$

The formula for the vector triple product has been used here. Notice that the constant of proportionality is the same as above.

Now consider the Clifford algebra formula,

$$
\begin{gathered}
\pi_{1} \pi_{2}-\pi_{2} \pi_{1}=2\left(n_{y 1} n_{z 2}-n_{z 1} n_{y 2}\right) e_{2} e_{3}+2\left(n_{z 1} n_{x 2}-n_{x 1} n_{z 2}\right) e_{3} e_{1}+2\left(n_{x 1} n_{y 2}-n_{y 1} n_{x 2}\right) e_{1} e_{2}+ \\
2\left(d_{2} n_{x 1}-d_{1} n_{x 2}\right) e_{1} e+2\left(d_{2} n_{y 1}-d_{1} n_{y 2}\right) e_{2} e+2\left(d_{2} n_{z 1}-d_{1} n_{z 2}\right) e_{3} e
\end{gathered}
$$

This is clearly proportional to the line determined by the planes. The constant of proportionality is the magnitude of $\mathbf{n}_{1} \times \mathbf{n}_{2}$, the sine of the angle between the planes. Hence, if the planes are parallel or anti-parallel the above expression yields zero. A condition for the planes to be parallel or anti-parallel is thus,

$$
\pi_{1} \pi_{2}-\pi_{2} \pi_{1}=0
$$

Otherwise, the line determined by the planes can be found by setting,

$$
\pi_{1} \pi_{2}-\pi_{2} \pi_{1}=\lambda, \quad \text { so that the line is } \quad \ell=\lambda / \sqrt{\lambda \lambda^{*}}
$$

Notice that, interchanging the order of the planes changes the sign of $\ell$. That is, the orientation of the line is reversed.

\subsection{A Line and a Plane}

Here we take a line,

$$
\ell=v_{x} e_{2} e_{3}+v_{y} e_{3} e_{1}+v_{z} e_{1} e_{2}+u_{x} e_{1} e+u_{y} e_{2} e+u_{z} e_{3} e
$$

and a plane,

$$
\pi=n_{x} e_{1}+n_{y} e_{2}+n_{z} e_{3}+d e
$$

Usually, a line and a plane will meet at a single point. Suppose this point is $\mathbf{r}$, then since the point lies on the plane we have $\mathbf{n} \cdot \mathbf{r}=d$. Also because the point lies on the line we have $\mathbf{r} \times \mathbf{v}=\mathbf{u}$. So consider the following triple product,

$$
\mathbf{n} \times(\mathbf{r} \times \mathbf{v})=\mathbf{n} \times \mathbf{u}=(\mathbf{n} \cdot \mathbf{v}) \mathbf{r}-d \mathbf{v}
$$

using the formula for the vector triple product. The intersection point is therefore proportional to,

$$
\mathbf{r} \propto \mathbf{n} \times \mathbf{u}+d \mathbf{v}
$$


and the constant of proportionality is $1 /(\mathbf{n} \cdot \mathbf{v})$.

A short computation shows that the Clifford algebra formula is given by,

$$
\begin{aligned}
& \ell \pi+\pi \ell=2\left(n_{x} v_{x}+n_{y} v_{y}+n_{z} v_{z}\right) e_{1} e_{2} e_{3}+ \\
& 2\left(n_{y} u_{z}-n_{z} u_{y}+d v_{x}\right) e_{2} e_{3} e+2\left(n_{z} u_{x}-n_{x} u_{z}+d v_{y}\right) e_{3} e_{1} e+2\left(n_{x} u_{y}-n_{y} u_{x}+d v_{z}\right) e_{1} e_{2} e
\end{aligned}
$$

The quantity, $\mathbf{n} \cdot \mathbf{v}$ only vanishes when the line is perpendicular to the plane's normal. Moreover,

$$
\ell \pi+\pi \ell=0
$$

gives the condition for the line to lie the plane. Otherwise, we get a unique point of intersection given by setting,

$$
\ell \pi+\pi \ell=\rho, \quad \text { so that the point is } \quad p=\rho / \sqrt{\rho \rho^{*}}
$$

Actually, for a practical computation we do not need to compute a square root since we can simply divide by the coefficient of $e_{1} e_{2} e_{3}$. The square root in the formula above is simply to avoid introducing another operation into the algebra and for consistency with later results.

\subsection{Three Planes}

Here we find the point determined by three planes. The planes will be written,

$$
\begin{aligned}
& \pi_{1}=n_{1 x} e_{1}+n_{1 y} e_{2}+n_{1 z} e_{3}+d_{1} e \\
& \pi_{2}=n_{2 x} e_{1}+n_{2 y} e_{2}+n_{2 z} e_{3}+d_{2} e \\
& \pi_{3}=n_{3 x} e_{1}+n_{3 y} e_{2}+n_{3 z} e_{3}+d_{3} e
\end{aligned}
$$

The point common to these three planes will be the solution to the system of linear equations,

$$
\begin{aligned}
& n_{1 x} x+n_{1 y} y+n_{1 z} z=d_{1} \\
& n_{2 x} x+n_{2 y} y+n_{2 z} z=d_{2} \\
& n_{3 x} x+n_{3 y} y+n_{3 z} z=d_{3}
\end{aligned}
$$

This is easily solved in terms of determinants. Now in the Clifford algebra we can take the alternating sum of the products of three planes,

$$
\begin{gathered}
\pi_{1} \pi_{2} \pi_{3}-\pi_{2} \pi_{1} \pi_{3}+\pi_{2} \pi_{3} \pi_{1}-\pi_{3} \pi_{2} \pi_{1}+\pi_{3} \pi_{1} \pi_{2}-\pi_{1} \pi_{3} \pi_{2}= \\
6\left|\begin{array}{lll}
n_{1 x} & n_{1 y} & n_{1 z} \\
n_{2 x} & n_{2 y} & n_{2 z} \\
n_{3 x} & n_{3 y} & n_{3 z}
\end{array}\right| e_{1} e_{2} e_{3}+6\left|\begin{array}{lll}
d_{1} & n_{1 y} & n_{1 z} \\
d_{2} & n_{2 y} & n_{2 z} \\
d_{3} & n_{3 y} & n_{3 z}
\end{array}\right| e_{2} e_{3} e+ \\
6\left|\begin{array}{lll}
n_{1 x} & d_{2} & n_{1 z} \\
n_{2 x} & d_{2} & n_{2 z} \\
n_{3 x} & d_{3} & n_{3 z}
\end{array}\right| e_{3} e_{1} e_{3}+6\left|\begin{array}{lll}
n_{1 x} & n_{1 y} & d_{1} \\
n_{2 x} & n_{2 y} & d_{2} \\
n_{3 x} & n_{3 y} & d_{3}
\end{array}\right| e_{1} e_{2} e
\end{gathered}
$$




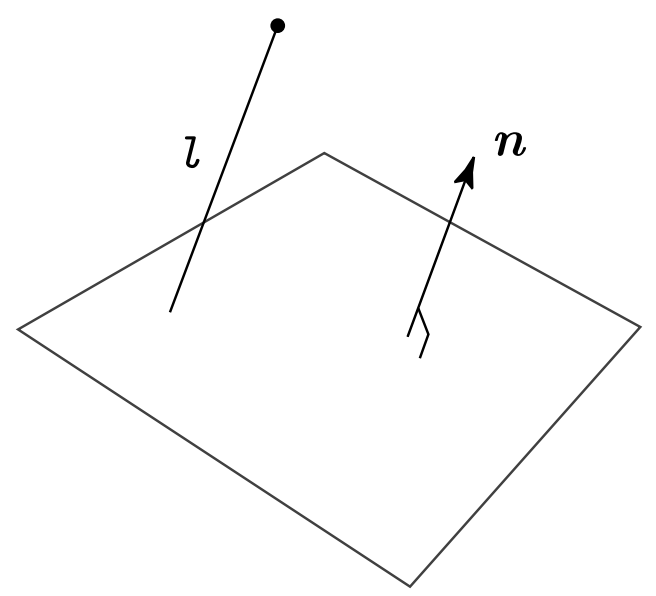

Figure 3: Distance from a Point to a Plane

Hence we may find the point of intersection by setting,

$$
\pi_{1} \pi_{2} \pi_{3}-\pi_{2} \pi_{1} \pi_{3}+\pi_{2} \pi_{3} \pi_{1}-\pi_{3} \pi_{2} \pi_{1}+\pi_{3} \pi_{1} \pi_{2}-\pi_{1} \pi_{3} \pi_{2}=\rho
$$

and then the point is,

$$
p=\rho / \sqrt{\rho \rho^{*}}
$$

Again, it is not really necessary to take a square root here, rather we can divide by the coefficient of $e_{1} e_{2} e_{3}$.

\subsection{A Point and a Plane}

Given a point,

$$
p=e_{1} e_{2} e_{3}+r_{x} e_{2} e_{3} e+r_{y} e_{3} e_{1} e+r_{z} e_{1} e_{2} e
$$

and a plane,

$$
\pi=n_{x} e_{1}+n_{y} e_{2}+n_{z} e_{3}+d e
$$

the minimum distance between the them lies along a normal to the plane and is given by, $l=\mathbf{n} \cdot \mathbf{r}-d$, see figure 3 . This quantity will be positive if the point is on the same side of the plane as the normal vector.

In terms of the Clifford algebra, we have,

$$
\pi p-p \pi=2 l e_{1} e_{2} e_{3} e
$$

That is,

$$
l=\frac{1}{2} \star(\pi p-p \pi)
$$

The condition for the point to lie on the plane is simply,

$$
\pi p-p \pi=0
$$


We can also find the line perpendicular to the plane and passing through the point. Clearly the direction of the line is the same as the normal vector to the plane and the moment of the line is given by $\mathbf{r} \times \mathbf{n}$. In the Clifford algebra we have,

$p \pi+\pi p=-2\left(n_{x} e_{2} e_{3}+n_{y} e_{3} e_{1}+n_{z} e_{1} e_{2}+\left(n_{z} r_{y}-n_{y} r_{z}\right) e_{1} e+\left(n_{z} r_{x}-n_{x} r_{z}\right) e_{2} e+\left(n_{x} r_{y}-n_{y} r_{x}\right) e_{3} e\right)$

\subsection{A Point and a Line}

Let the point be,

$$
p=e_{1} e_{2} e_{3}+r_{x} e_{2} e_{3} e+r_{y} e_{3} e_{1} e+r_{z} e_{1} e_{2} e
$$

and the line,

$$
\ell=v_{x} e_{2} e_{3}+v_{y} e_{3} e_{1}+v_{z} e_{1} e_{2}+u_{x} e_{1} e+u_{y} e_{2} e+u_{z} e_{3} e
$$

As long as the point does not lie on the line then the point and line will determine a plane. Now if $\mathbf{q}$ is a point on the line we have,

$$
(\mathbf{q}-\mathbf{r}) \times \mathbf{v}=\mathbf{u}-\mathbf{r} \times \mathbf{v}=s \mathbf{n}
$$

where $s$ is the distance from the point to the line and $\mathbf{n}$ is the unit normal to the plane containing the point and the line. To find the distance of the plane to the origin we can take the dot product of the normal with a point $\mathbf{r}$ on the plane,

$$
(\mathbf{u}-\mathbf{r} \times \mathbf{v}) \cdot \mathbf{r}=\mathbf{u} \cdot \mathbf{r}=s d
$$

In the Clifford algebra we have,

$\ell p-p \ell=2\left(u_{x}-r_{y} v_{z}+r_{z} v_{y}\right) e_{2} e_{3} e+2\left(u_{y}-r_{z} v_{x}-r_{x} v_{z}\right) e_{3} e_{1} e+2\left(u_{z}-r_{x} v_{y}-r_{y} v_{x}\right) e_{1} e_{2} e$

Hence, the condition for the point to lie on the line is simply,

$$
\ell p-p \ell=0
$$

To produce the plane determined by the line and the point we need the Hodge star operation. In the Clifford algebra we have,

$$
\begin{aligned}
& (\star \ell)(\star p)+(\star p)(\star \ell)=-2\left(r_{x} u_{x}+r_{y} u_{y}+r_{z} u_{z}\right) e_{1} e_{2} e_{3}- \\
& \quad 2\left(u_{x}-r_{y} v_{z}+r_{z} v_{y}\right) e_{2} e_{3} e-2\left(u_{y}-r_{z} v_{x}-r_{x} v_{z}\right) e_{3} e_{1} e-2\left(u_{z}-r_{x} v_{y}-r_{y} v_{x}\right) e_{1} e_{2} e
\end{aligned}
$$

So if we set,

$$
(\star \ell)(\star p)+(\star p)(\star \ell)=\sigma, \quad \text { then the plane is } \quad \pi=(\star \sigma) / \sqrt{(\star \sigma)(\star \sigma)^{*}}
$$

Notice that the orientation of the plane is determined by the direction of the line and the position of the point.

Finally here, consider the expression,

$$
p \ell+\ell p=-2 v_{x} e_{1}-2 v_{y} e_{2}-2 v_{z} e_{3}-2\left(r_{x} v_{x}+r_{y} v_{y}+r_{z} v_{z}\right) e
$$

This, up to an overall constant is the plane perpendicular to the line $\ell$ but passing through the point $p$. 


\subsection{Pairs of Lines}

Here, we look at the disposition of a pair of lines,

$$
\begin{aligned}
& \ell_{1}=v_{x 1} e_{2} e_{3}+v_{y 1} e_{3} e_{1}+v_{z 1} e_{1} e_{2}+u_{x 1} e_{1} e+u_{y 1} e_{2} e+u_{z 1} e_{3} e \\
& \ell_{2}=v_{x 2} e_{2} e_{3}+v_{y 2} e_{3} e_{1}+v_{z 2} e_{1} e_{2}+u_{x 2} e_{1} e+u_{y 2} e_{2} e+u_{z 2} e_{3} e
\end{aligned}
$$

We have several things to consider here. First the angle between the lines $\alpha$, this is given (up to a sign) by, $\mathbf{v}_{1} \cdot \mathbf{v}_{2}=\cos \alpha$. Then there is the distance between the lines, this is the minimum distance which is measured along the common perpendicular. Let $s$ be this distance and suppose $\mathbf{r}_{1}$ and $\mathbf{r}_{2}$ are the respective points on the two lines which meet the common perpendicular. Now $\mathbf{r}_{2}-\mathbf{r}_{1}$ is a vector of length $s$ along the perpendicular which is parallel to the vector $\mathbf{v}_{2} \times \mathbf{v}_{1}$. Hence we have,

$$
s \sin \alpha=\left(\mathbf{r}_{2}-\mathbf{r}_{1}\right) \cdot \mathbf{v}_{2} \times \mathbf{v}_{1}=\mathbf{v}_{1} \cdot \mathbf{u}_{2}+\mathbf{u}_{1} \cdot \mathbf{v}_{2}
$$

This gives the distance $s$, which is always positive and hence the sign of the angle between the line.

In the Clifford algebra we have,

$$
\begin{aligned}
& \ell_{1} \ell_{2}+\ell_{2} \ell_{1}=-2\left(v_{x 1} v_{x 2}+v_{y 1} v_{y 2}+v_{z 1} v_{z 2}\right)+ \\
& 2\left(v_{x 1} u_{x 2}+v_{y 1} u_{y 2}+v_{z 1} u_{z 2}+u_{x 1} v_{x 2}+u_{y 1} v_{y 2}+u_{z 1} v_{z 2}\right) e_{1} e_{2} e_{3} e
\end{aligned}
$$

That is,

$$
\frac{1}{2}\left(\ell_{1} \ell_{2}^{*}+\ell_{2} \ell_{1}^{*}\right)=\cos \alpha-s \sin \alpha e_{1} e_{2} e_{3} e
$$

Remember that $\ell^{*}=-\ell$. This means that if $1 / 2\left(\ell_{1} \ell_{2}^{*}+\ell_{2} \ell_{1}^{*}\right)= \pm 1$ then the lines $\ell_{1}$ and $\ell_{2}$ are parallel or anti-parallel. On the other hand if $1 / 2\left(\ell_{1} \ell_{2}^{*}+\ell_{2} \ell_{1}^{*}\right)=0$ then the lines meet and are perpendicular.

Finally here, we look at the line perpendicular to a pair of lines. From above we have that the line $\ell$ perpendicular to a pair of lines $\ell_{1}$ and $\ell_{2}$ must satisfy the linear equations,

$$
\ell_{1} \ell^{*}+\ell \ell_{1}^{*}=0 \quad \text { and } \quad \ell_{2} \ell^{*}+\ell \ell_{2}^{*}=0
$$

It is simple to check that the commutator expression $\ell_{1} \ell_{2}-\ell_{2} \ell_{1}$ satisfies both equations, unfortunately it is not usually a line, that is it does not satisfy $\ell \ell^{*}=1$. In the Clifford algebra the commutator is,

$$
\begin{gathered}
\ell_{1} \ell_{2}-\ell_{2} \ell_{1}=2\left(v_{y 1} v_{z 2}-v_{z 1} v_{y 2}\right) e_{2} e_{3}+2\left(v_{z 1} v_{x 2}-v_{x 1} v_{z 2}\right) e_{3} e_{1}+2\left(v_{x 1} v_{y 2}-v_{y 1} v_{x 2}\right) e_{1} e_{2}+ \\
2\left(v_{y 1} u_{z 2}-v_{z 1} u_{y 2}+u_{y 1} v_{z 2}-u_{z 1} v_{y 2}\right) e_{1} e+ \\
2\left(v_{z 1} u_{x 2}-v_{x 1} u_{z 2}+u_{z 1} v_{x 2}-u_{x 1} v_{z 2}\right) e_{2} e+ \\
2\left(v_{x 1} u_{y 2}-v_{y 1} u_{x 2}+u_{x 1} v_{y 2}-u_{y 1} v_{x 2}\right) e_{3} e
\end{gathered}
$$

Now consider a specific example, say $\ell_{1}=e_{2} e_{3}$, this is a line along the $x$-axis, and $\ell_{2}=\cos \alpha e_{2} e_{3}+\sin \alpha e_{1} e_{1}-s \sin \alpha e_{1} e+s \cos \alpha e_{2} e$, which is at an angle $\alpha$ to the first line but displaced $s$ units along the $z$-axis. The line perpendicular to this pair of lines is clearly along the $z$-axis. The commutator $1 / 2\left(\ell_{1} \ell_{2}-\ell_{2} \ell_{1}\right)$ is,

$$
\frac{1}{2}\left(\ell_{1} \ell_{2}-\ell_{2} \ell_{1}\right)=\sin \alpha e_{1} e_{2}+s \cos \alpha e_{3} e=\left(\sin \alpha+s \cos \alpha e_{1} e_{2} e_{3} e\right) e_{1} e_{2}
$$


Suppose now we have an arbitrary pair of lines in space, we can always find a rigid body motion $g$ which will translate the lines to the configuration given above. The transform of a line is given by $\ell^{\prime}=g \ell g^{*}$ and hence the commutator transforms as $1 / 2\left(\ell_{1}^{\prime} \ell_{2}^{\prime}-\ell_{2}^{\prime} \ell_{1}^{\prime}\right)=1 / 2 g\left(\ell_{1} \ell_{2}-\ell_{2} \ell_{1}\right) g^{*}$. The coefficient $\left(\sin \alpha+s \cos \alpha e_{1} e_{2} e_{3} e\right)$ is easily seen to be invariant with respect to rigid transformations. Hence, for a general pair of lines we have the result,

$$
\frac{1}{2}\left(\ell_{1} \ell_{2}-\ell_{2} \ell_{1}\right)=\left(\sin \alpha+s \cos \alpha e_{1} e_{2} e_{3} e\right) \ell
$$

where $\alpha$ is the angle between the lines $s$ their minimum distance and $\ell$ the common perpendicular line.

The commutator defined above plays a central role in the Lie algebra of the group of rigid body motions, see [6].

\subsection{Pairs of Points}

Let the two points be,

$$
\begin{aligned}
& p_{1}=e_{1} e_{2} e_{3}+x_{1} e_{2} e_{3} e+y_{1} e_{3} e_{1} e+z_{1} e_{1} e_{2} e \\
& p_{2}=e_{1} e_{2} e_{3}+x_{2} e_{2} e_{3} e+y_{2} e_{3} e_{1} e+z_{2} e_{1} e_{2} e
\end{aligned}
$$

The line joining the two points in the direction from $p_{1}$ to $p_{2}$ can be found from the formula,

$$
\begin{aligned}
\left(\star p_{1}\right)\left(\star p_{2}\right)- & \left(\star p_{2}\right)\left(\star p_{1}\right)=2\left(y_{1} z_{2}-z_{1} y_{2}\right) e_{2} e_{3}+2\left(z_{1} x_{2}-x_{1} z_{2}\right) e_{3} e_{1}+ \\
& 2\left(x_{1} y_{2}-y_{1} x_{2}\right) e_{1} e_{2}+2\left(x_{2}-x_{1}\right) e_{1} e+2\left(y_{2}-y_{1}\right) e_{2} e+2\left(z_{2}-z_{1}\right) e_{3} e
\end{aligned}
$$

So the line can be found by setting,

$$
\left(\star p_{1}\right)\left(\star p_{2}\right)-\left(\star p_{2}\right)\left(\star p_{1}\right)=\lambda, \quad \text { and thus the line is } \quad \ell=(\star \lambda) / \sqrt{(\star \lambda)(\star \lambda)^{*}}
$$

The distance between a pair of points can be found from the relation,

$$
p_{1} p_{2}-p_{2} p_{1}=2\left(x_{1}-x_{2}\right) e_{1} e+2\left(y_{1}-y_{2}\right) e_{2} e+2\left(z_{1}-z_{2}\right) e_{3} e
$$

However, a shorter method results from the relation,

$$
\left(p_{2}-p_{1}\right)\left(\star\left(p_{2}-p_{1}\right)\right)=\left(\left(x_{2}-x_{1}\right)^{2}+\left(y_{2}-y_{1}\right)^{2}+\left(z_{2}-z_{1}\right)^{2}\right) e_{1} e_{2} e_{3} e
$$

\subsection{Three Points}

Finally here, we look at the plane determined by three points, Let the three points be $\mathbf{r}_{1} \mathbf{r}_{2}$ and $\mathbf{r}_{3}$ represented by the Clifford algebra elements,

$$
\begin{aligned}
& p_{1}=e_{1} e_{2} e_{3}+x_{1} e_{2} e_{3} e+y_{1} e_{3} e_{1} e+z_{1} e_{1} e_{2} e \\
& p_{2}=e_{1} e_{2} e_{3}+x_{2} e_{2} e_{3} e+y_{2} e_{3} e_{1} e+z_{2} e_{1} e_{2} e \\
& p_{3}=e_{1} e_{2} e_{3}+x_{3} e_{2} e_{3} e+y_{3} e_{3} e_{1} e+z_{3} e_{1} e_{2} e
\end{aligned}
$$


The normal to the plane determined by the points will be proportional to,

$$
\mathbf{n} \propto\left(\mathbf{r}_{2}-\mathbf{r}_{1}\right) \times\left(\mathbf{r}_{3}-\mathbf{r}_{1}\right)=\mathbf{r}_{2} \times \mathbf{r}_{3}+\mathbf{r}_{3} \times \mathbf{r}_{1}+\mathbf{r}_{1} \times \mathbf{r}_{2}
$$

The distance from the origin can be found by taking the scalar product of the normal with any point on the plane,

$$
d=\mathbf{n} \cdot \mathbf{r}_{1} \propto \mathbf{r}_{1} \cdot\left(\mathbf{r}_{2} \times \mathbf{r}_{3}\right)
$$

In the Clifford algebra we look at the alternating sum,

$$
\begin{aligned}
& \left(\star p_{1}\right)\left(\star p_{2}\right)\left(\star p_{3}\right)-\left(\star p_{2}\right)\left(\star p_{1}\right)\left(\star p_{3}\right)+\left(\star p_{2}\right)\left(\star p_{3}\right)\left(\star p_{1}\right)- \\
& \left(\star p_{3}\right)\left(\star p_{2}\right)\left(\star p_{1}\right)+\left(\star p_{3}\right)\left(\star p_{1}\right)\left(\star p_{2}\right)-\left(\star p_{1}\right)\left(\star p_{3}\right)\left(\star p_{2}\right)= \\
& 6\left(y_{1} z_{2}-z_{1} y_{2}+y_{2} z_{3}-z_{2} y_{3}+z_{1} y_{3}-y_{1} z_{3}\right) e_{2} e_{3} e+ \\
& 6\left(z_{1} x_{2}-x_{1} z_{2}+z_{2} x_{3}-x_{2} z_{3}+z_{3} x_{1}-x_{3} z_{1}\right) e_{3} e_{1} e+ \\
& 6\left(x_{1} y_{2}-y_{1} x_{2}+x_{2} y_{3}-y_{2} x_{3}+y_{1} x_{3}-x_{1} y_{3}\right) e_{1} e_{2} e+ \\
& 6\left(x_{1} z_{2} y_{3}-x_{1} y_{2} z_{3}+y_{1} x_{2} z_{3}-y_{1} z_{2} y_{3}+z_{1} y_{2} x_{3}\right) e_{1} e_{2} e_{3}
\end{aligned}
$$

Hence, if we set,

$$
\begin{aligned}
\left(\star p_{1}\right)\left(\star p_{2}\right)\left(\star p_{3}\right)- & \left(\star p_{2}\right)\left(\star p_{1}\right)\left(\star p_{3}\right)+\left(\star p_{2}\right)\left(\star p_{3}\right)\left(\star p_{1}\right)- \\
& \left(\star p_{3}\right)\left(\star p_{2}\right)\left(\star p_{1}\right)+\left(\star p_{3}\right)\left(\star p_{1}\right)\left(\star p_{2}\right)-\left(\star p_{1}\right)\left(\star p_{3}\right)\left(\star p_{2}\right)=\sigma
\end{aligned}
$$

then the plane will be given by,

$$
\pi=(\star \sigma) / \sqrt{(\star \sigma)(\star \sigma)^{*}}
$$

Notice that the condition for the point to be collinear will be,

$$
\sigma=0
$$

\subsection{Summary}

In this summary section the main results given above are collected together in a more convenient form. First of all the incidence relations,

- A point $p$ lies on a line $\ell$ if and only if $p \ell^{*}+\ell p^{*}=0$.

- A point $p$ lies on a plane $\pi$ if and only if $p \pi^{*}+\pi p^{*}=0$.

- A line $\ell$ lies in a plane $\pi$ if and only if $\ell \pi^{*}+\pi \ell^{*}=0$.

Notice how the inclusion of the conjugate makes these relations look uniform. Recall that, $p^{*}=p$ while $\ell^{*}=-\ell$ and $\pi^{*}=-\pi$.

In general two lines in 3-D are skew, the condition for them to be coplanar is, $\star\left(\ell_{1} \ell_{2}^{*}+\ell_{2} \ell_{1}^{*}\right) e=0$. That is, the coefficient of $e_{1} e_{2} e_{3} e$ in the expression $\left(\ell_{1} \ell_{2}^{*}+\ell_{2} \ell_{1}^{*}\right)$ is zero. Notice that, for lines to be coplanar, either they meet at a point or they are parallel. Parallel lines are often thought of as meeting "at infinity".

Next we look at the distance relation between pairs of elements. In most cases only positive distances make sense, hence nothing is lost if the square of the distance is found. 
- The distance $s$, between a pair of points $p_{1}$ and $p_{2}$ can be found from,

$$
s^{2}=\frac{1}{4}(\star \mu)(\star \mu)^{*} \quad \text { where } \quad \mu=p_{1} p_{2}-p_{2} p_{1}
$$

Recall that, in this case we also have the simpler formula, $s^{2}=\star\left(\left(p_{1}-p_{2}\right) \star\right.$ $\left.\left(p_{1}-p_{2}\right)\right)$.

- The perpendicular distance $s$, from a point $p$ to a line $\ell$ can be found from,

$$
s^{2}=\frac{1}{4}(\star v)(\star v)^{*} \quad \text { where } \quad v=\ell p-p \ell
$$

- The perpendicular distance $l$, from a point $p$ to a plane $\pi$ is

$$
l=\frac{1}{2} \star(\pi p-p \pi)
$$

This is a directed distance, the quantity is positive if the point is on the same side as the normal to the plane and negative if it is on the other side of the plane. Notice however, that this formula is consistent with the ones given immediately above since,

$$
l^{2}=\frac{1}{4}(\star \xi)(\star \xi)^{*} \quad \text { where } \quad \xi=\pi p-p \pi
$$

The intersection between pairs of linear elements is another linear element sometimes referred to as the meet of the two original elements. The formulae for the meet of general elements are given by,

- The point determined by the intersection of a line $\ell$ and a plane $\pi$ is,

$$
p=\rho / \sqrt{\rho \rho^{*}} \text { where } \rho=\ell \pi^{*}-\pi \ell^{*}
$$

Recall that this can be done more simply by dividing $\rho$ by the coefficient of $e_{1} e_{2} e_{3}$.

- The line determined by the intersection of a pair of planes $\pi_{1}$ and $\pi_{2}$ is,

$$
\ell=\lambda / \sqrt{\lambda \lambda^{*}} \text { where } \lambda=\pi_{1} \pi_{2}^{*}-\pi_{2} \pi_{1}^{*}
$$

- The point determined by the intersection of three planes $\pi_{1}, \pi_{2}$ and $\pi_{3}$ is given by,

$$
p=\rho / \sqrt{\rho \rho^{*}} \text { where } \rho=\sum_{i j k} \varepsilon_{i j k} \pi_{i} \pi_{j} \pi_{k}
$$

Again we can do without the square root by dividing by the coefficient for the term $e_{1} e_{2} e_{3}$. The symbol $\varepsilon_{i j k}$ in the above represents the alternating tensor, this has a value of +1 if $i j k$ is an even permutation of 123 and -1 if the permutation is odd, and in all other cases its value is 0 .

The join of a pair of linear elements is the linear span of the subspaces determined by the elements. The results we have found for the joins of elements are, 
- The line determined by a pair of points $p_{1}$ and $p_{2}$ is given by,

$$
\ell=(\star \lambda) / \sqrt{(\star \lambda)(\star \lambda)^{*}} \text { where } \lambda=\left(\star p_{1}\right)\left(\star p_{2}\right)^{*}-\left(\star p_{2}\right)\left(\star p_{1}\right)^{*}
$$

- The plane determined by a point $p$ and a line $\ell$ is given by,

$$
\pi=(\star \sigma) / \sqrt{(\star \sigma)(\star \sigma)^{*}} \text { where } \sigma=(\star \ell)(\star p)^{*}-(\star p)(\star \ell)^{*}
$$

- The plane determined by three points $p_{1}, p_{2}$ and $p_{3}$ is given by,

$$
\pi=(\star \sigma) / \sqrt{(\star \sigma)(\star \sigma)^{*}} \quad \text { where } \quad \sigma=\sum_{i j k} \varepsilon_{i j k}\left(\star p_{1}\right)\left(\star p_{2}\right)\left(\star p_{3}\right)
$$

where we have used the alternating tensor $\varepsilon_{i j k}$ again.

The relations between pairs of lines are slightly different in character from the results given above. This is because they involve the notion of perpendicularity. Given a pair of lines $\ell_{1}$ and $\ell_{2}$ we have,

$$
\begin{aligned}
& \frac{1}{2}\left(\ell_{1} \ell_{2}^{*}+\ell_{2} \ell_{1}^{*}\right)=\left(\cos \alpha-s \sin \alpha e_{1} e_{2} e_{3} e\right) \\
& \frac{1}{2}\left(\ell_{1} \ell_{2}-\ell_{2} \ell_{1}\right)=\left(\sin \alpha+s \cos \alpha e_{1} e_{2} e_{3} e\right) \ell^{\perp}
\end{aligned}
$$

where, $\alpha$ is the angle between the lines, $s$ the minimum distance between the lines and $\ell^{\perp}$ the line perpendicular to both lines. From these result it is possible to derive conditions for the lines to be parallel or perpendicular.

There are two final results on perpendicularity,

- The plane $\pi^{\perp}$, perpendicular to a line $\ell$ and passing through a point $p$ is given by,

$$
\pi^{\perp}=\frac{1}{2}\left(p \ell^{*}-\ell p^{*}\right)
$$

- The line $\ell^{\perp}$, perpendicular to a plane $\pi$ and passing through a point $p$ is given by,

$$
\ell^{\perp}=\frac{1}{2}\left(p \pi^{*}-\pi p^{*}\right)
$$

\section{Examples}

\subsection{Visibility}

In many graphics applications the following problem is of some importance. Suppose we have a pair of lines in space, $\ell_{1}$ and $\ell_{2}$. In general these line will be skew, but if we project them onto an image plane their projections will meet, usually at a point, see figure 4 . The problem is to compute the coordinates of this crossing point. To simplify the computations we suppose that the centre of projection, or focus point is located at 


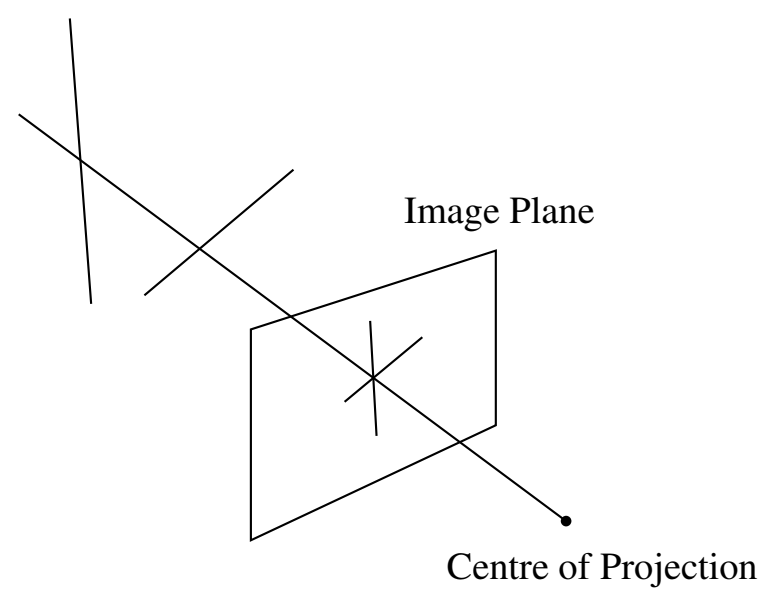

Figure 4: The Apparent Crossing of Two Lines

the origin, this point will be labelled, $p_{0}=e_{1} e_{2} e_{3}$. The plane of projection will be be taken to be the plane,

$$
\pi=e_{3}+f e
$$

That is, a plane parallel to the $x y$-plane but displaced $f$ units in the $z$-direction.

The two lines will be given by,

$$
\begin{aligned}
& \ell_{1}=v_{x 1} e_{2} e_{3}+v_{y 1} e_{3} e_{1}+v_{z 1} e_{1} e_{2}+u_{x 1} e_{1} e+u_{y 1} e_{2} e+u_{z 1} e_{3} e \\
& \ell_{2}=v_{x 2} e_{2} e_{3}+v_{y 2} e_{3} e_{1}+v_{z 2} e_{1} e_{2}+u_{x 2} e_{1} e+u_{y 2} e_{2} e+u_{z 2} e_{3} e
\end{aligned}
$$

To find the crossing point of the projections of these lines we find the line through the centre of projection which meets both lines. This line will be the meet of the two planes formed by the centre of projection and each of the lines in space. The plane formed by the first line and the centre of projection is given by,

$$
\left(\star \pi_{1}\right) \propto\left(\star \ell_{1}\right)\left(\star p_{0}\right)+\left(\star p_{0}\right)\left(\star \ell_{1}\right)=2 u_{x 1} e_{2} e_{3} e+2 u_{y 1} e_{3} e_{1} e+2 u_{z 1} e_{1} e_{2} e
$$

hence,

$$
\pi_{1} \propto-\left(u_{x 1} e_{1}+u_{y 1} e_{2}+u_{z 1} e_{3}\right)
$$

and a similar expression can be found for the plane formed by the second line. The meet of these two planes can be computed as follows,

$\ell \propto\left(\pi_{1} \pi_{2}-\pi_{2} \pi_{1}\right)=\left(u_{y 1} u_{z 2}-u_{z 1} u_{y 2}\right) e_{2} e_{3}+\left(u_{z 1} u_{x 2}-u_{x 1} u_{z 2}\right) e_{3} e_{1}+\left(u_{x 1} u_{y 2}-u_{y 1} u_{x 2}\right) e_{1} e_{2}$

The constants of proportionality will be zero if either line passes through the centre of projection or if both lines project to the same line in the image plane. In these cases the solution is not defined. However, in all other cases we don't need to find the constant of proportionality. We could find the image coordinates by intersecting $\ell$ with the image plane, however the problem is so simple that the more usual method of similar triangle 
is easier. The image point has coordinates $\left(x_{I}, y_{I}, f\right)$ and lies in the direction of the position vector given by, $\left(u_{y 1} u_{z 2}-u_{z 1} u_{y 2}, u_{z 1} u_{x 2}-u_{x 1} u_{z 2}, u_{x 1} u_{y 2}-u_{y 1} u_{x 2}\right)$. Hence, the image coordinates of the crossing point $\left(x_{I}, y_{I}\right)$, are given by,

$$
x_{I}=f \frac{\left(u_{y 1} u_{z 2}-u_{z 1} u_{y 2}\right)}{\left(u_{x 1} u_{y 2}-u_{y 1} u_{x 2}\right)}, \quad \text { and } \quad y_{I}=f \frac{\left(u_{z 1} u_{x 2}-u_{x 1} u_{z 2}\right)}{\left(u_{x 1} u_{y 2}-u_{y 1} u_{x 2}\right)}
$$

These simple formulas demonstrate the utility of specifying a line using its direction and moment vector, the solution is given in terms of the components of the vector product of the moment vectors of the two lines. To reinforce the point we can look at what happens under a change of viewpoint.

Suppose the change of viewpoint is given by a rigid body motion $g$, that is both the centre of projection and the image plane undergo the same transformation. Usually the result will be required in the local coordinates fixed to the image plane. Hence rather than transform the centre of projection and image plane we can apply $g^{*}$, the inverse of $g$, to the lines. Assume that the transformation $g^{*}$ is given by a rotation $R$ and a translation $\mathbf{t}$, as in section 2.3 above, the vector product of the moment vectors is now,

$$
\mathbf{u}_{1}^{\prime} \times \mathbf{u}_{2}^{\prime}=\left(R \mathbf{u}_{1}+\mathbf{t} \times R \mathbf{v}_{1}\right) \times\left(R \mathbf{u}_{2}+\mathbf{t} \times R \mathbf{v}_{2}\right)
$$

Finally here, it is important to know whether or not the apparent crossing can be seen. That is, do both the points on the lines corresponding to the crossing point in the projection, lie in front of the image plane? To find the point where the ray through the crossing point meets $\ell_{1}$ we can intersect $\ell_{1}$ with the plane $\pi_{2}$,

$$
\begin{aligned}
& p_{1} \propto \ell_{1} \pi_{2}+\pi_{2} \ell_{1} \propto\left(v_{x 1} u_{x 2}+v_{y 1} u_{y 2}+v_{z 1} u_{z 2}\right) e_{1} e_{2} e_{3}+ \\
& \quad\left(u_{z 1} u_{y 2}-u_{y 1} u_{z 2}\right) e_{2} e_{3} e+\left(u_{x 1} u_{z 2}-u_{z 1} u_{x 2}\right) e_{3} e_{1} e+\left(u_{y 1} u_{x 2}-u_{x 1} u_{y 2}\right) e_{1} e_{2} e
\end{aligned}
$$

The constant of proportionality here is clearly $\mathbf{v}_{1} \cdot \mathbf{u}_{2}$. Now, if we assume for the sake of argument, that the point can be seen if it is on the opposite side of the image plane $\left(\pi=e_{3}+f e\right)$ to the origin then we will get,

$$
\pi p_{1}-p_{1} \pi=\frac{2}{\mathbf{v}_{1} \cdot \mathbf{u}_{2}}\left(u_{y 1} u_{x 2}-u_{x 1} u_{y 2}-f\left(\mathbf{v}_{1} \cdot \mathbf{u}_{2}\right)\right) e_{1} e_{2} e_{3} e
$$

The coefficient is positive, and hence the point visible, when,

$$
u_{y 1} u_{x 2}-u_{x 1} u_{y 2}>f\left(\mathbf{v}_{1} \cdot \mathbf{u}_{2}\right)
$$

For the apparent crossing to be visible both this point and the corresponding point on $\ell_{2}$ must be visible.

\subsection{Assembly}

The following example is taken more or less directly from [2]. The problem is to insert a pair of pegs into two holes. Each peg or hole determines a line in space hence the problem becomes: Find group elements which transform a line $\ell_{1}$ to $\ell_{1}^{\prime}$ and simultaneously map the line $\ell_{2}$ to $\ell_{2}^{\prime}$, where $\ell_{1}$ and $\ell_{1}^{\prime}$ are the lines determined by the first peg and hole and $\ell_{2}$ and $\ell_{2}^{\prime}$ come from the second peg and hole. 
Now we may express the problem as a pair of equations in the group elements $g$,

$$
\begin{aligned}
& \ell_{1}^{\prime}=g \ell_{1} g^{*} \\
& \ell_{2}^{\prime}=g \ell_{2} g^{*}
\end{aligned}
$$

A simple manipulation turns these equations into a system of homogeneous linear equations for the coefficients of $g$,

$$
\begin{aligned}
& \ell_{1}^{\prime} g-g \ell_{1}=0 \\
& \ell_{2}^{\prime} g-g \ell_{2}=0
\end{aligned}
$$

Each of these Clifford algebra equations represents 4 equations for the eight unknown coefficients of the group element $g$. This gives 8 linear equations in all which, together with the quadratic condition $g g^{*}=1$ for the group elements means that the system is over-determined. Hence, we seek conditions for a solution to exist. Take the first of the equations and post multiply it by the conjugate of the second to give, after a little rearrangement,

$$
g \ell_{1} \ell_{2}^{*} g^{*}=\ell_{1}^{\prime} \ell_{2}^{\prime *}
$$

If we perform the same operation in the other order and add the result to the above we get,

$$
g\left(\ell_{1} \ell_{2}^{*}+\ell_{2} \ell_{1}^{*}\right) g^{*}=\left(\ell_{1} \ell_{2}^{*}+\ell_{2} \ell_{1}^{*}\right)=\left(\ell_{1}^{\prime} \ell_{2}^{\prime *}+\ell_{2}^{\prime} \ell_{1}^{*}\right)
$$

Since, the combination $\ell_{1} \ell_{2}^{*}+\ell_{2} \ell_{1}^{*}$ is invariant under the action of the group. The relation derived above tells us that if a solution exists then the angle and separation of the two pairs of lines must be the same.

Now suppose that two different solutions exist $g_{1} \neq g_{2}$. If we set $g=g_{1} g_{2}^{*}$ then is not difficult to see that we must have,

$$
\ell_{1} g=g \ell_{1} \quad \text { and } \quad \ell_{2} g=g \ell_{2}
$$

The condition $g_{1} \neq g_{2}$, implies that $g \neq 1$. The general solution to one of the above equations, say the first, is a linear combination of the elements $1, e_{1} e_{2} e_{3} e, \ell_{1}$ and $\ell_{1} e_{1} e_{2} e_{3} e$. If $\ell_{1} \neq \ell_{2}$ then a simultaneous solution to the two equations cannot contain $\ell_{1}$. For a non-trivial solution we must have $\ell_{1} e_{1} e_{2} e_{3} e=\ell_{2} e_{1} e_{2} e_{3} e$. This implies that the lines are parallel since it tells us that the direction vectors along the lines are the same, remember that peg 1 must be aligned with hole 1 and peg 2 with hole 2 .

\subsection{Stereo Vision}

Suppose we have a pair of cameras looking at the same scene. As in section 5.1 above, each camera can be specified by a centre of projection, or focus, and an image plane. An object point in the scene determines a line through the focus of each camera. The image point is the point where that line meets the image plane of the particular camera. Now, the correspondence problem in stereo vision is the problem of identifying pairs of image points, one from each camera, which correspond to the same object point in the original scene. This problem is greatly simplified by finding the epipolar line. Suppose we know the image point in the right camera, the original object point must have been 


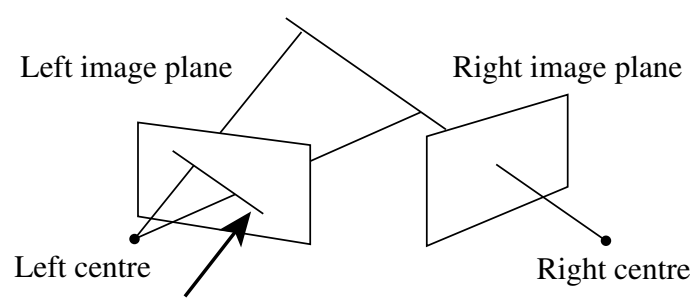

Epipolar line

(a)

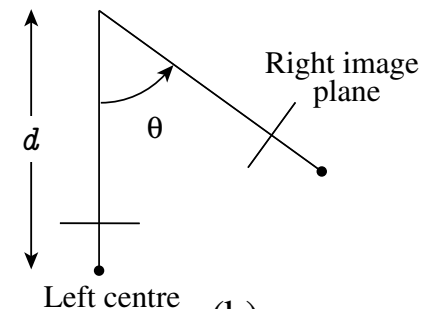

(b)

Figure 5: (a) The Epipolar Line in a General Set-Up (b) A Particular Example of a Stereo System

a point lying on the line joining this right image point with the right focus, let us call this line $\ell_{R}$. The set of possible image points in the left camera is now restricted to intersections of the left image plane with lines through the left focus which meet $\ell_{R}$. This set of possibilities for the left image point comprise a line in the left image plane which is known as the epipolar line see figure 5(a).

In the standard case where the cameras are identical and their relative positions are given by a pure translation perpendicular to the optical axes of the cameras, the problem of finding the epipolar line is trivial and can be found in any standard robot vision text. See [7, Chap. 7], for example. If the cameras are in general position the problem is much harder, especially if we are forced to rely on schoolbook geometry. However, using the methods developed above we can give a simple method for the most general situation.

Let $p_{1}$ and $p_{2}$ be the right and left focus points respectively and suppose $p_{3}$ is the image point in the right plane. Now we find the plane formed by these three points and intersect this plane with the left image plane $\pi_{L}$. The resulting line is the epipolar line corresponding to the right image point $p_{3}$. Notice that in practice we do not need the plane given by the three points but only the algebra element proportional to the plane. This is because normalisation will take place at the end of the computation anyway. So we compute,

$$
\sigma=\sum \varepsilon_{i j k}\left(\star p_{i}\right)\left(\star p_{j}\right)\left(\star p_{k}\right)
$$

and then,

$$
\lambda=\pi_{L}(\star \sigma)-(\star \sigma) \pi_{L}
$$

and the epipolar line is $\ell=\lambda / \sqrt{\lambda \lambda^{*}}$.

A slight complication to this very simple scheme is that usually points and lines in both the left and right image planes will be specified in terms of local (image) coordinates. Suppose that the left camera is located so that the focus coincides with the origin and the image plane is perpendicular to the $z$-axis. With this choice of coordinates the global coordinates correspond with the image coordinates of the left camera and we have,

$$
p_{2}=e_{1} e_{2} e_{3}
$$


Next suppose that the group element $g$ takes the right camera to the standard position described above. If $p_{R}=e_{1} e_{2} e_{3}$ and $p_{I}=e_{1} e_{2} e_{3}+x_{R} e_{2} e_{3} e+y_{R} e_{3} e_{1} e+f_{R} e_{1} e_{2} e$ are respectively the position of the right focus and image points given in local coordinates ( $f_{R}$ is the focal distance from the focus to the image plane). In the global coordinate system these points become,

$$
p_{1}=g^{*} p_{R} g, \quad \text { and } \quad p_{3}=g^{*} p_{I} g
$$

Using these values in the procedure outlined above will yield the epipolar line in coordinates local to the left camera.

Often the line in the left image will be required in the form of the standard equation, $a x+b y=c$. The epipolar line in the left image plane will be given by,

$$
\ell=v_{x} e_{2} e_{3}+v_{y} e_{3} e_{1}+f v_{y} e_{1} e+f v_{y} e_{2} e+\left(x_{L} v_{y}-y_{L} v_{x}\right) e_{3} e
$$

Let $(x, y)$ be a point on the line in image coordinates, the equation of the line expresses the fact that the projection of the point onto a direction perpendicular to the line is constant. That is, $v_{y} x-v_{x} y=c$. We can see from this that $c$ is the $z$-component of the moment of the line:

$$
v_{y} x-v_{x} y=u_{z}
$$

To make the above clearer we can look at a particular example. Consider the case of two identical cameras, whose optical axes lie in a plane and whose focal lengths are both $f$. Further, suppose that the distance between the focus of either camera and the meeting point of the optical axes is $d$, see figure 5(b). This is a fairly common configuration, familiar from normal human vision. The angle between the optical axes is known as the angle of vergence and will be denoted $\theta$ here. The computation for the equation of the epipolar line proceeds as follows.

$$
\begin{aligned}
g & =\cos \frac{\theta}{2}+\sin \frac{\theta}{2} e_{3} e_{1}-d \sin \frac{\theta}{2} \\
p_{1}= & g^{*} e_{1} e_{2} e_{3} g \\
& =e_{1} e_{2} e_{3}+d \sin \theta e_{2} e_{3} e+d(1-\cos \theta) e_{1} e_{2} e
\end{aligned}
$$




$$
\begin{aligned}
& \left.d(1-\cos \theta) \sin \theta) e_{3} e_{1} e-y_{R} \sin \theta e_{1} e_{2} e\right) \\
& \pi_{L}=e_{3}+f e \\
& \lambda=\pi_{L}(\star \sigma)-(\star \sigma) \pi_{L} \\
& =B e_{2} e_{3}-A e_{3} e_{1}+A f e_{1} e+B f e_{2} e+C f e_{3} e \\
& A=12 d y_{R}(1-\cos \theta) \\
& \left.B=12 d\left(x_{R}(1-\cos \theta)+f \sin \theta+d(1-\cos \theta) \sin \theta\right)\right) \\
& C=12 d y_{R} \sin \theta
\end{aligned}
$$

Hence the equation of the epipolar line in the left camera corresponding to the point $\left(x_{R}, y_{R}\right)$ in the right camera is,

$$
-A x_{L}-B y_{L}=C
$$

or more explicitly,

$$
\left.y_{R}(1-\cos \theta) x_{L}+\left(x_{R}(1-\cos \theta)+f \sin \theta+d(1-\cos \theta) \sin \theta\right)\right) y_{L}+y_{R} \sin \theta=0
$$

Notice, that we could fix $d$ and $\theta$ to get a result which simply relates the coordinates of the point in the right image to the epipolar line in the left image. The above result would also be useful for more general stereo vision systems with actuated cameras.

\section{Concluding Remarks}

Some of the results found above will be familiar from Clifford's original biquaterion algebra, [5]. However, by staying with the original Clifford algebra we are able to include relations concerning points and planes.

The relations we have found are not particularly efficient from a computational point of view. By careful study it may be possible to improve this situation by not computing terms which will eventually cancel. For manual computations, these terms can be used to check for errors. As mentioned in the introduction, however, this work is intended for symbolic computation.

The Clifford algebra for points lines and planes presented above, can be extended in a reasonably simple way to represent flags, see [6, Chap. 10]. A flag is a nested sequence of linear elements, for example a pointed line is a line containing a distinguished point. From the above, it is clear that we can represent a pointed line by an algebra element of the form, $p+\ell$, where $p$ is a homogeneous element of grade 3 satisfying $p p^{*}=1$, that is a point, and $\ell$ is homogeneous of grade 2 satisfying $\ell \ell^{*}=1$, that is a line. To ensure that the point lies on the line we must have $p \ell^{*}+\ell p^{*}=0$. Notice that this shows that the space of all pointed lines is an algebraic variety. This has important consequences for the study of the geometry of the space. There are other spaces 
of partial flags: pointed planes and lined planes and also complete flags, consisting of a point on a line in a plane. The complete flags were called 'soma' by Study, [8]. These flag spaces are useful when looking at problems involving simple mechanical joints. For example, a pointed line can be used to represent a hinge or revolute joint while a lined plane can represent a sliding or prismatic joint. These ideas can then be applied in a fairly straightforward manner to problems in robot kinematics, in particular the inverse kinematics of serial manipulators, see [9].

Finally, in other work on Clifford algebra, notably [3], much is made of the maximum/minimum problems like finding least squares solutions to systems of constraint equations. A geometrical approach to such problems would be to differentiate with respect to vectors tangent to the group of rigid body motions. In the Clifford algebra we can represent such derivatives as commutators with elements of the Lie algebra of the group or rigid motion. This Lie algebra turns out to be precisely the space of homogeneous element of grade 2 in the Clifford algebra. Unfortunately, there is not enough space to elaborate on this here.

\section{Acknowledgement}

I wish to thank Ralph Martin many helpful comments on this work.

\section{References}

[1] A. Bowyer, J.H. Davenport, P. Milne, J. Padget, and A.F. Wallis. A geometric algebra system. In J. Woodwark, editor, Geometric Reasoning, Oxford Science Publications, pages 1-27, Oxford, 1989. Oxford: Clarendon Press.

[2] O.E. Ruiz and P.M. Ferreira. Algebraic geometry and group theory in geometric constraint satisfaction for computer-aided design and assembly planning. IIE Transactions, 28:281-294, 1996.

[3] C.J.L. Doran J. Lasenby, W. J. Fitzgerald and A.N. Lasenby. New geometric methods for computer vison: an application to structure and motion estimation. Int. J. Comp. Vision, 36(3):191-213, 1998.

[4] I.R. Porteous. Topological Geometry. Cambridge University Press, Cambridge, second edition, 1981.

[5] W.K. Clifford. Preliminary sketch of biquaternions. Proceedings of the London Mathematical Society, iv(64/65):381-395, 1873.

[6] J.M. Selig. Geometrical Methods in Robotics. Springer Verlag, New York, 1996.

[7] V.S. Nalwa. A Guided Tour of Computer Vision. Addison Wesley, Reading, Mass, 1993.

[8] E. Study. von den Bewegungen und Umlegungen. Math. Ann., 39:441-566, 1891. 
[9] J.M. Selig. Robot kinematics and flags. In E. Bayro-Corrochano and G. Sobczyk, editors, Geometric Algebra: A Geometric approach to Computer Vision, Quantum and Neural Computing, Robotics and Engineering. Kluwer Academic Publishers, To Appear. 


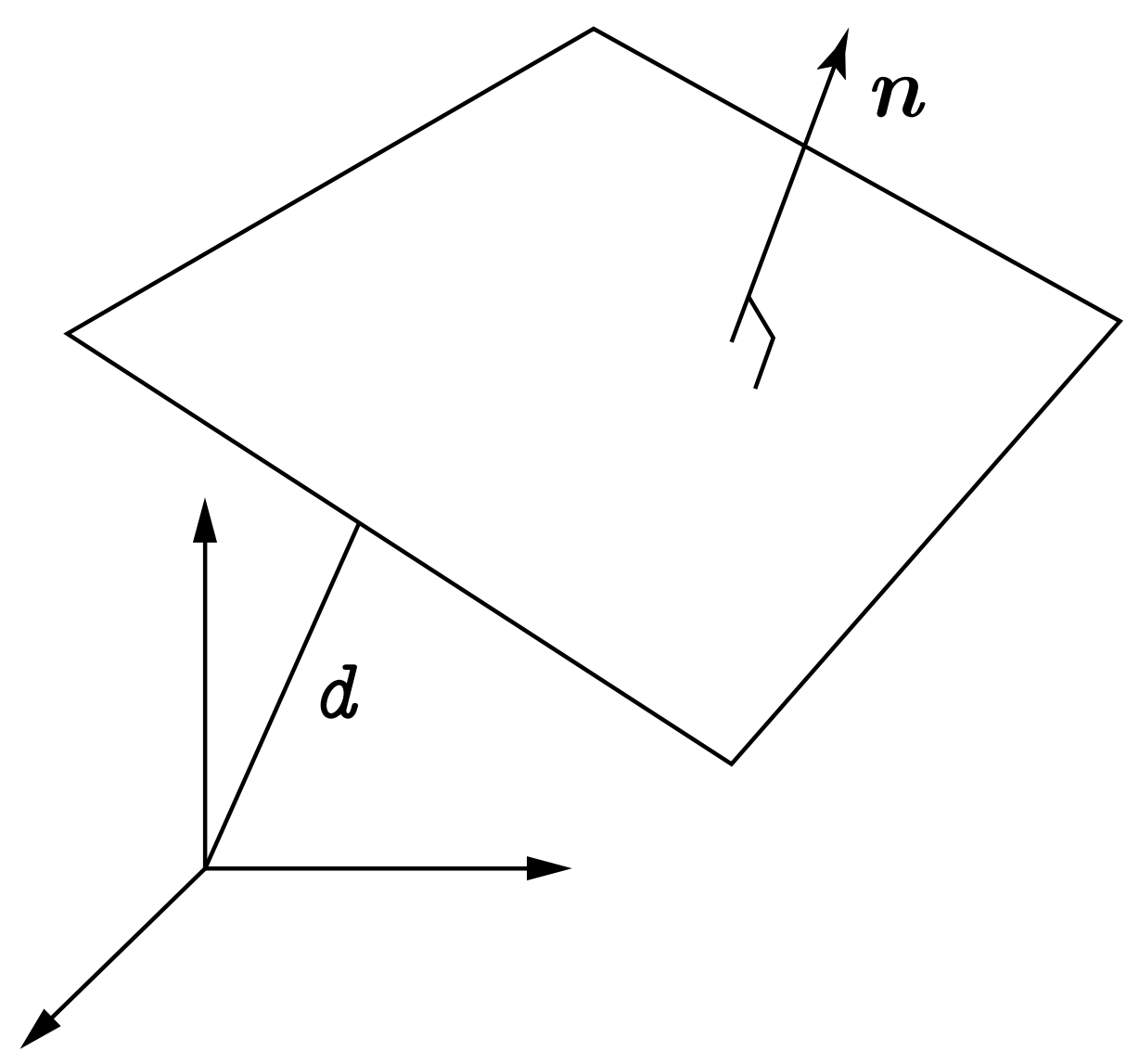

A Plane 


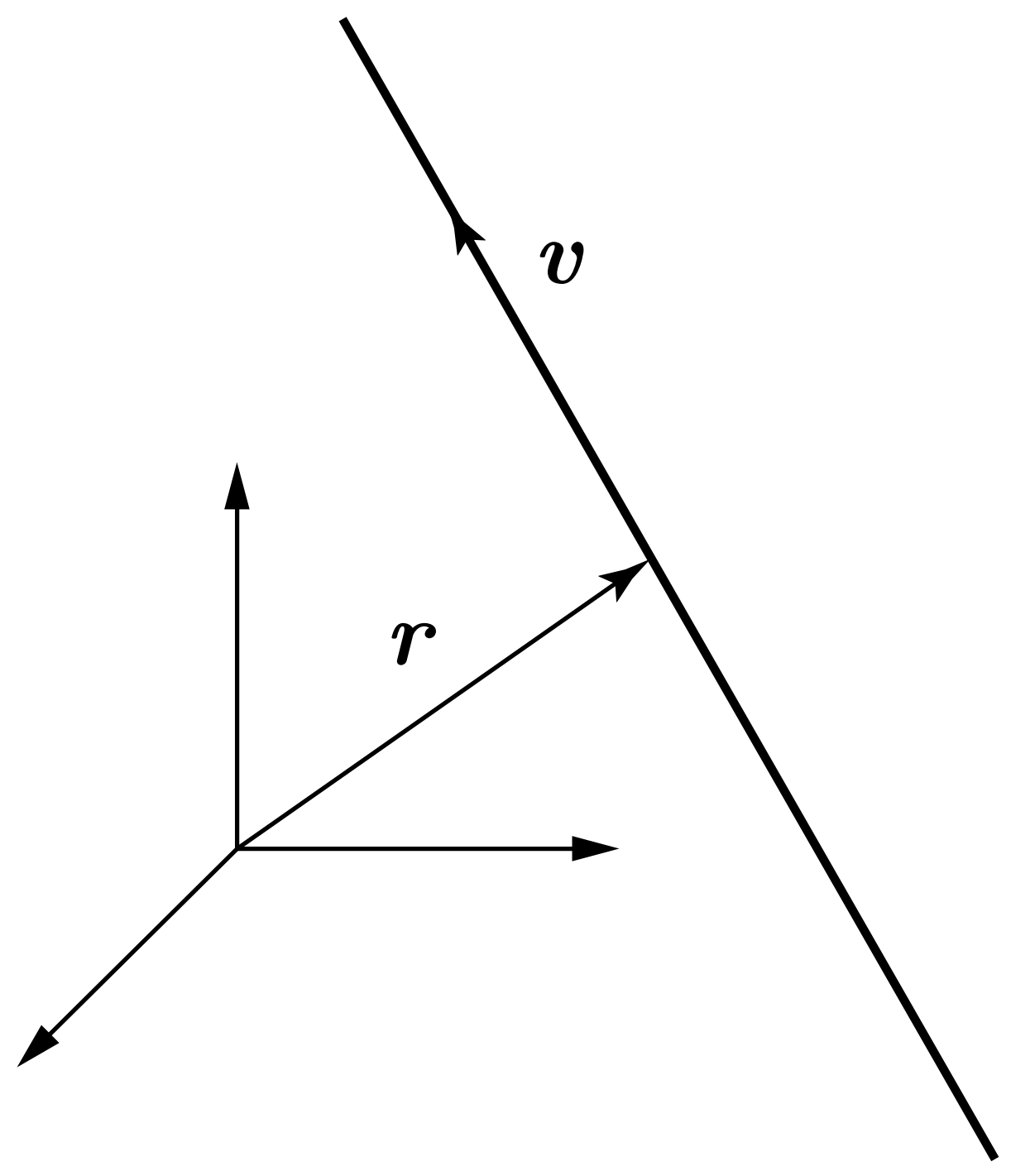

A Line in Space 


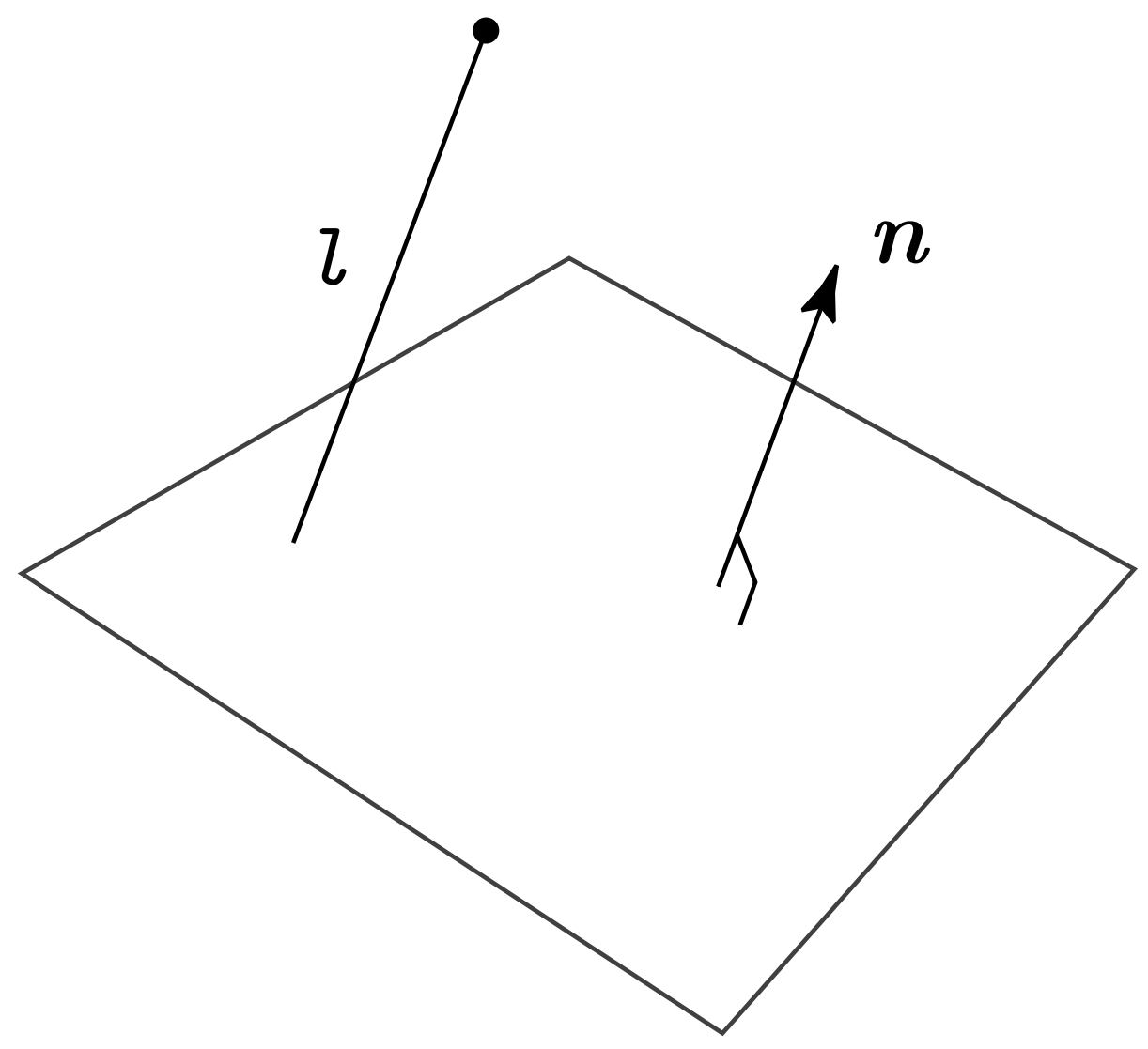

Distance from a Point to a Plane 


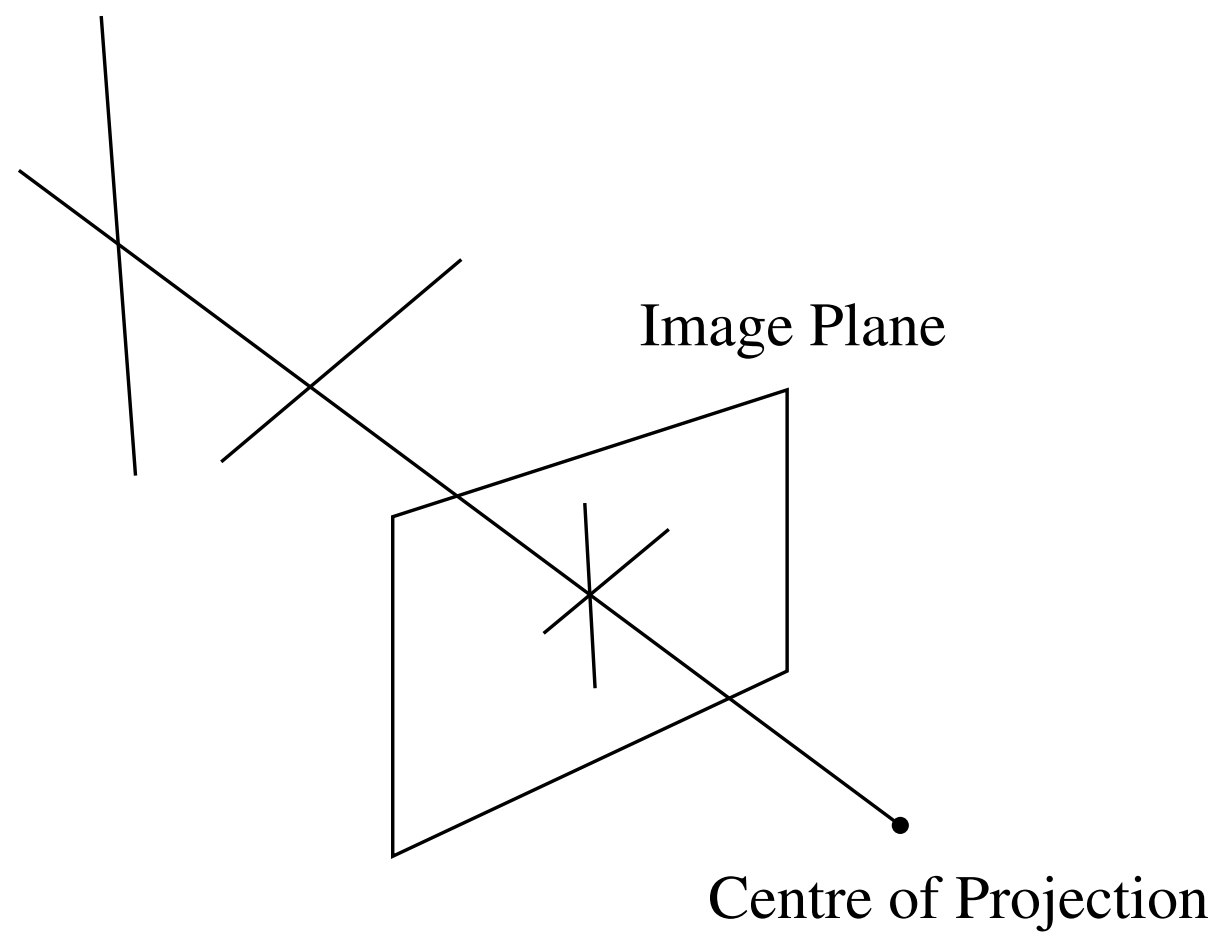

The Apparent Crossing of Two Lines 

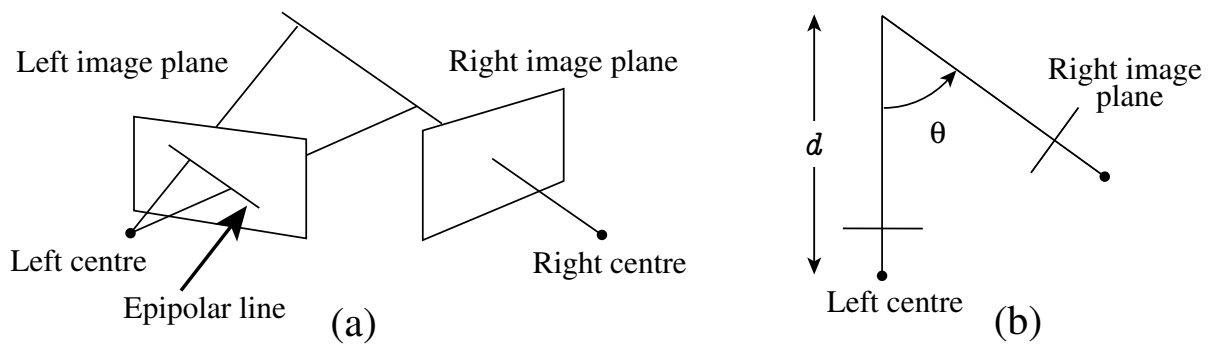

(a) The Epipolar Line in a General Set-Up (b) A Particular Example of a Stereo System 


\section{Figure Captions}

1. A Plane

2. A Line in Space

3. Distance from a Point to a Plane

4. The Apparent Crossing of Two Lines

5. (a) The Epipolar Line in a General Set-Up (b) A Particular Example of a Stereo System 